Article

\title{
Chlamydiaphage $\varphi$ CPG1 Capsid Protein Vp1 Inhibits Chlamydia trachomatis Growth via the Mitogen-Activated Protein Kinase Pathway
}

\author{
Yuanli Guo, Rui Guo, Quan Zhou, Changgui Sun, Xinmei Zhang, Yuanjun Liu \\ and Quanzhong Liu *
}

Dermatology and Venereology Department, Tianjin Medical University General Hospital, No. 154 Anshan Road, Heping District, Tianjin 300052, China; lvninle@sina.com (Y.G.); 13902052977@163.com (R.G.); peter-pf.liu@cn.ey.com (Q.Z.); 2008021327@tmu.edu.cn (C.S.); hongheifeiying@sina.com (X.Z.); liuyuanjun1980@163.com (Y.L.)

* Correspondence: liuquanzhong@medmail.com.cn; Tel./Fax: 86-022-60363131

Academic Editor: Rob Lavigne

Received: 30 December 2015; Accepted: 11 April 2016; Published: 14 April 2016

\begin{abstract}
Chlamydia trachomatis is the most common cause of curable bacterial sexually transmitted infections worldwide. Although the pathogen is well established, the pathogenic mechanisms remain unclear. Given the current challenges of antibiotic resistance and blocked processes of vaccine development, the use of a specific chlamydiaphage may be a new treatment solution. $\varphi$ CPG1 is a lytic phage specific for Chlamydia caviae, and shows over $90 \%$ nucleotide sequence identity with other chlamydiaphages. Vp1 is the major capsid protein of $\varphi$ CPG1. Purified Vp1 was previously confirmed to inhibit Chlamydia trachomatis growth. We here report the first attempt at exploring the relationship between Vp1-treated C. trachomatis and the protein and gene levels of the mitogen-activated/extracellular regulated protein kinase (MAPK/ERK) pathway by Western blotting and real-time PCR, respectively. Moreover, we evaluated the levels of pro-inflammatory cytokines interleukin (IL)-8 and IL-1 by enzyme-linked immunosorbent assay after Vp1 treatment. After $48 \mathrm{~h}$ of incubation, the p-ERK level of the Vp1-treated group decreased compared with that of the Chlamydia infection group. Accordingly, ERK1 and ERK2 mRNA expression levels of the Vp1-treated group also decreased compared with the Chlamydia infection group. IL-8 and IL-1 levels were also decreased after Vp1 treatment compared with the untreated group. Our results demonstrate that the inhibition effect of the chlamydiaphage $\varphi$ CPG1 capsid protein Vp1 on C. trachomatis is associated with the MAPK pathway, and inhibits production of the pro-inflammatory cytokines IL- 8 and IL- 1 . The bacteriophages may provide insight into a new signaling transduction mechanism to influence their hosts, in addition to bacteriolysis.
\end{abstract}

Keywords: Chlamydia trachomatis; mitogen-activated protein kinase pathway; chlamydiaphage; capsid protein Vp1; azithromycin

\section{Introduction}

Chlamydia trachomatis is the most common cause of curable bacterial sexually transmitted infection worldwide because of their prevalence and potentially devastating reproductive consequences, including pelvic inflammatory disease, infertility, and ectopic pregnancy. The number of chlamydialinfected citizens reached 1,422,976 in the United States in 2012 [1]. In China, the prevalence of chlamydial infection has also increased, and was reported to be 2.6 and 2.1 per 100 individuals for women and men, respectively [2]. Although the basic biology of the pathogen is well established, the pathogenic mechanisms are still unclear. Even worse, the condition of antibiotics resistance is becoming more and more serious. Therefore, further exploration of the Chlamydia infection mechanisms is sorely 
needed to develop new treatment methods to overcome the current public health challenges. Given the condition of a blocked process of developing a vaccine for Chlamydia trachomatis, use of a specific chlamydiaphage may be a new solution.

Chlamydiae have an obligate intracellular developmental cycle that alternates between the infectious elementary body and the replicative reticulate body. Six bacteriophages have been isolated from the Chlamydiae: Chp1, Chp2, Chp3, Chp4, $\varphi$ CPG1, and $\varphi$ CPAR39. All of the chlamydiaphages share similar features; they are small, icosahedral $\mathrm{T}=1$ particles containing circular, single-stranded DNA genomes, and molecular characterization revealed that they belong to the virus family Microviridae [3-5]. $\varphi$ CPG1 is a lytic phage specific to Chlamydia caviae, which is a natural parasite of the guinea pig. The genome of $\varphi$ CPG1 contains five open reading frames, which encode the capsid proteins $\mathrm{Vp} 1, \mathrm{Vp} 2$, and Vp3. Capsid proteins, especially Vp1, play essential roles in adhesion and invasion. Our research group has compared the Vp1 of all Chlamydia phages found until now and recovered nearly $98 \%$ comparability among them. Even though a specific bacteriophage of $C$. trachomatis has not yet been detected, the high homology among the known chlamydiaphages allows for the reasonable assumption of cross-reaction among species. The phage host range is specified by a peptidic loop of $\mathrm{Vp} 1$, and sequence variation within the loop is consistent with the observed overlapping host ranges of the phages [6]. Vp1 also plays an important role in the interaction between Chlamydia and its host cell. Our research group successfully expressed and purified Vp1, which was incubated with $C$. trachomatis and confirmed to inhibit its growth [7]. However, the specific mechanism underlying this inhibition effect has not yet been explored.

The mechanisms of $C$. trachomatis infection remain mysterious. Most research conducted in this field thus far has considered the inflammation-induced pathological damage [8,9]. The C. trachomatis-induced inflammation reaction is mediated by signaling pathways in the host cell that are triggered after infection, including the mitogen-activated protein kinase (MAPK) pathway, Janus-activated kinase-signal transducer and activator of transcription (JAK/STAT) pathway, and nuclear factor-kappa B (NF- $\mathrm{kB}$ ) pathway, among others. In particular, the MAPK pathway plays a major role in post-infection signal transduction, and the growth of $C$. trachomatis depends on the MAPK/ERK (mitogen-activated protein kinase / extracellular regulated protein kinases) pathway. During the course of incubation in the host, $C$. trachomatis enters into its own growth cycle, which induces increased phosphorylation levels of ERK protein in the host cell. Once the growth cycle is blocked with U0126, a specific inhibitor of MEK, the infection rate decreases, the major outer membrane protein (MOMP) level of $C$. trachomatis is reduced, and the phosphorylation level of ERK $1 / 2$ of the host is downregulated [10]. These facts reveal that $C$. trachomatis modulates the host MEK/ERK pathway and relies on it for its growth. In response to the increase in p-ERK, the host cell will release pro-inflammatory factors such as interleukin (IL)-1, IL-8, and tumor necrosis factor (TNF)- $\alpha$ [11]. IL-1 and IL-8 are considered the most significant factors contributing to host cell inflammatory injury in C. trachomatis infection $[12,13]$.

Azithromycin has been the recommended antibiotic for treatment of $C$. trachomatis infection according to the guideline. Previous studies reported that azithromycin inhibits cytokine production, which may contribute to its therapeutic effect in the treatment of chronic $C$. trachomatis infection [14]. In addition, phosphorylation of ERK was found to be inhibited after azithromycin treatment in epithelial cells obtained from women with recurrent infection [15]. Traditionally, bacteriolysis is considered the main mechanism by which a bacteriophage inhibits the growth of its host. However, there may be alternative mechanisms that have not yet been explored, which could provide useful insights for the development of new treatments. In the present study, we aimed to address the following remaining questions related to the mechanism of Chlamydia growth inhibition by Vp1. In particular, we investigated whether Vp1 might exert its inhibition effect via the MAPK pathway similar to azithromycin, and compared the inhibition effect of Vp1 and azithromycin. 


\section{Materials and Methods}

\subsection{McCoy Cell Culture}

McCoy cells (mouse fibroblast L cell origin) were purchased from the Chinese Academy of Medical Sciences, and stored in the Tianjin Institute of Sexually Transmitted Diseases, China. They were grown in minimal essential medium supplemented with $10 \%$ fetal bovine serum for Chlamydia culture. When the cells were distributed compactly and uniformly as a single layer, they were transferred to 6-well plates for continuous incubation and Chlamydia infection.

\subsection{C. trachomatis Infection}

The monolayer McCoy cells were pretreated with $30 \mu \mathrm{g} / \mathrm{mL}$ of DEAE-D for $30 \mathrm{~min}$ to increase the susceptibility of infection. The C. trachomatis strain used in this study was the E serotype strain that is maintained in our laboratory. The strain was pretreated with two freeze-thawing cycles, oscillation, and centrifugation at $500 \times \mathrm{g}$ for $5 \mathrm{~min}$. The plates were further centrifuged at $500 \times \mathrm{g}$ at $32{ }^{\circ} \mathrm{C}$ for $1 \mathrm{~h}$ to facilitate Chlamydia adhesion. After $2 \mathrm{~h}$, the wells of the plate were overlaid with culture medium containing $1 \mathrm{mg} / \mathrm{L}$ cycloheximide, and incubated for $44-48 \mathrm{~h}$. The majority of cells were collected in transport culture medium, and the remaining cells were fixed with methanol and stained with iodine dye to observe the residual inclusions to verify that the infective rate was over $90 \%$.

\subsection{Vp1 Expression, Identification, and Purification}

The stored E. coli bacteria that contained Vp1-pET30a(+) [16] were incubated in kanamycinresistant Luria-Bertani medium for 12-16 h. The bacteria were amplified in the shaker and Vp1 expression was induced with $0.03 \mathrm{mM}$ isopropyl- $\beta$-D-thiogalactopyranoside (IPTG) at $30{ }^{\circ} \mathrm{C}$ for $3 \mathrm{~h}$ until reaching the logarithmic phase (OD 0.6-0.8 in $600 \mathrm{~nm}$ ). The bacterial solution was centrifuged and the sediment was treated with $4 \mathrm{mg} / \mathrm{mL}$ lysozyme and 3\% Triton-X100. The suspension was ultrasonicated and centrifuged. The sediment was blended in PBS with $6 \mathrm{~mol} / \mathrm{L}$ urea and identified by sodium dodecyl sulfate-polyacrylamide gel electrophoresis (SDS-PAGE). The purification of Vp1 was achieved with the PAGE recovery method, renaturated by dialysis and quantified for further use. LPS (Lipopolysaccharides) was neutralized less than $0.1 \mathrm{EU} / \mathrm{mL}$ by ToxinEraser ${ }^{\mathrm{TM}}$ endotoxin removal resin kit from GenScript (Piscataway, NJ, USA). Vp1 was confirmed without toxic effect on McCoy cells by MTT (3-(4,5-dimethyl-2-thiazolyl)-2,5-diphenyl-2-H-tetrazolium bromide) method.

\subsection{Treatments and Experimental Design}

The McCoy cells were incubated with or without C. trachomatis, Vp1 and azithromycin, and classified into 6 experimental groups (Table 1). C. trachomatis was infected at $3 \times 10^{5} \mathrm{IFU} / \mathrm{mL}$ after purification and quantification. The inoculation loads of $\mathrm{Vp} 1$ and azithromycin were $60 \mu \mathrm{g} / \mathrm{mL}$ and $25 \mu \mathrm{g} / \mathrm{mL}$, according to previous reports ${ }^{12}$ and the results of preliminary experiments. After $48 \mathrm{~h}$, the inclusions were counted under the microscope and stained with iodine dye.

Table 1. Design groups for treatment.

\begin{tabular}{ccccccc}
\hline & Group C & Group C + V & Group C + A & Group V & Group A & Group M \\
\hline C. trachomatis & $\sqrt{ }$ & $\sqrt{ }$ & $\sqrt{ }$ & & & \\
Vp1 & & $\sqrt{ }$ & $\sqrt{ }$ & & & \\
Azithromycin & & $\sqrt{ }$ & $\sqrt{ }$ & $\sqrt{ }$ & $\sqrt{ }$ & $\sqrt{ }$ \\
McCoy cell & $\sqrt{ }$ & $\sqrt{ }$ & & \\
\hline
\end{tabular}

Notes: C: C. trachomatis, V: Vp1, A: Azithromycin, M: McCoy cell. 


\subsection{Western Blotting}

The cells were incubated at $0,12 \mathrm{~h}, 24 \mathrm{~h}, 36 \mathrm{~h}$, or $48 \mathrm{~h}$, according to treatment, and the cellular proteins was extracted by the RIPA (Radio Immunoprecipitation Assay) method. The total proteins were separated by SDS-PAGE. The polyvinylidene fluoride (PVDF) membrane (Millipore Immobilon-P, Darmstadt, Germany). The monoclonal antibodies of p-ERK1/2 and total ERK1/2 (Cell Signaling Technology, Danvers, MA, USA) were added to the relevant treatment groups at a dilution of 1:1000. The corresponding secondary antibodies (Cell Signaling Technology) were added at dilutions of 1:5000-1:10000. The PVDF membrane was exposed and photographed after development, and the grey value ratio was used for evaluation determined with Gel-Pro Analyzer Software (Media Cybernetics, Rockville, MD, USA). The p-ERK1/2 level was standardized by the grey value ratio of ERK $1 / 2$. The experiment was repeated three times and the results are presented as the mean and standard deviation.

\subsection{Real-Time PCR}

The PCR protocols were performed according to the methods described by Wang and Seed [17]. In brief, cells in Groups M, C, C + V, and C + A were incubated for $48 \mathrm{~h}$ in 6-well plates, and the cells were dissociated and centrifuged at $500 \times \mathrm{g}$ for collection according to the treatment groups. Total RNA was extracted by the TRNzol method and its purity and concentration were verified on a spectrophotometer. Reverse transcription was carried out with the PrimeScript RT reagent Kit (Qiagen, Hilden, Germany) with gDNA Eraser (Qiagen) for RT-PCR. The primers were synthesized by Invitrogen (Carlsbad, CA, USA) (Table 2). The PCR program was carried out at $95^{\circ} \mathrm{C} 30 \mathrm{~s},\left(95^{\circ} \mathrm{C} 5 \mathrm{~s}\right.$, $\left.60{ }^{\circ} \mathrm{C} 40 \mathrm{~s}\right) \times 45$ with SYBR $\circledast$ Premix Ex Taq ${ }^{\mathrm{TM}}$ II (Tli RNaseH Plus) (Takara Bio, Dalian, China), ROX plus (Takara Bio) on an ABI 7500 system (Applied Biosystems, Foster City, CA, USA). The results were quantitatively analyzed based on Actin by the $2^{-\Delta \Delta c t}$ method.

Table 2. The primer sequences used for PCR.

\begin{tabular}{cclc}
\hline Gene & & \multicolumn{1}{c}{ Primer Sequences $\left(\mathbf{5}^{\prime}\right.$ to $\mathbf{3}^{\prime}$ ) } & Products Size (bp) \\
\hline \multirow{2}{*}{ Mapk1 (ERK2) } & F & GTATTCTTGGATCTCCATCACAGG & \multirow{2}{*}{246} \\
& R & TGGGCTCATCACTTGGGTCA & \\
\multirow{2}{*}{ Mapk3 (ERK1) } & F & CAAACAAGCGCATCACAGTAGA & 113 \\
& R & CAGCTCCATGTCGAAGGTGAAT & \\
Actin & F & GCCTTCCTTCTTGGGTAT & 97 \\
& R & GGCATAGAGGTCTTTACGG & \\
\hline
\end{tabular}

\subsection{IL-1 and IL-8 Enzyme-Linked Immunosorbent Assay}

The supernatants of treated cells from the different groups were collected at various time points of incubation $(0,12 \mathrm{~h}, 24 \mathrm{~h}, 36 \mathrm{~h}, 48 \mathrm{~h})$ and subjected to enzyme-linked immunosorbent assay for detection of cytokine (IL-1 and IL-8) production levels. The experiment was repeated three times and the results are presented as the mean with standard deviation.

\subsection{Data Analysis}

All of the data were analyzed with SPSS 17.0 software (IBM, Armonk, NY, USA) according to the statistics. The difference was considered statistically significant if the $p$-value was less than 0.05 .

\section{Results}

\subsection{ERK1/2 Phosphorylation Increased throughout C. trachomatis Growth}

During the culture of cells in Group C, which only contained the Chlamydia, the expression of $\mathrm{p}$-ERK1/2 protein showed a tendency to gradually increase (Figure 1A). The p-ERK1/2 levels substantially decreased from 0 to $12 \mathrm{~h}$, as determined by the decrease in the grey value ratio from 
$0.662 \pm 0.039$ to $0.022 \pm 0.005$. This initial decrease was likely due to the presence of $1 \mathrm{mg} / \mathrm{mL}$ cycloheximide in the culture medium, which is an antibody against actinomycetes and inhibits protein synthesis. However, in the subsequent period of Chlamydia culture, $12-48 \mathrm{~h}$, the expression of p-ERK1/2 gradually increased again, accompanied with an increase in the grey value ratio from $0.022 \pm 0.005$ to $0.542 \pm 0.078$. This result indicates that the Chlamydia activates ERK1/2 phosphorylation, confirming that $C$. trachomatis growth relies on the MEK/ERK pathway. The change in p-ERK1/2 levels over time was statistically significant (Figure 2A).

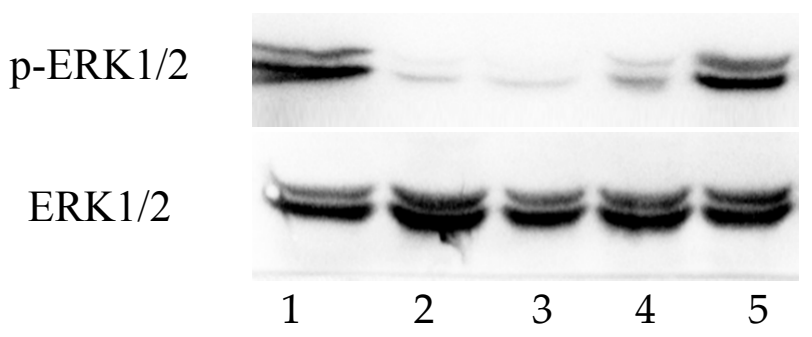

(A)

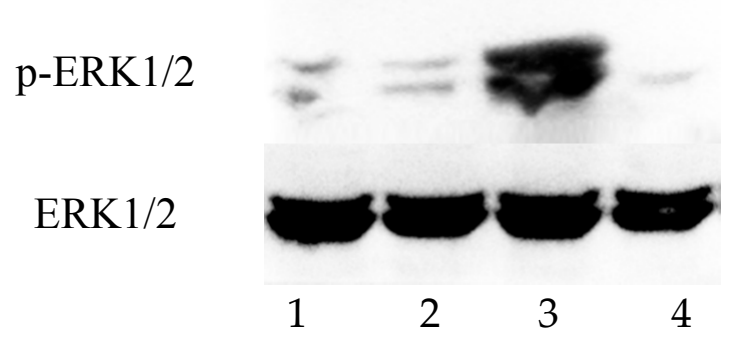

(B)
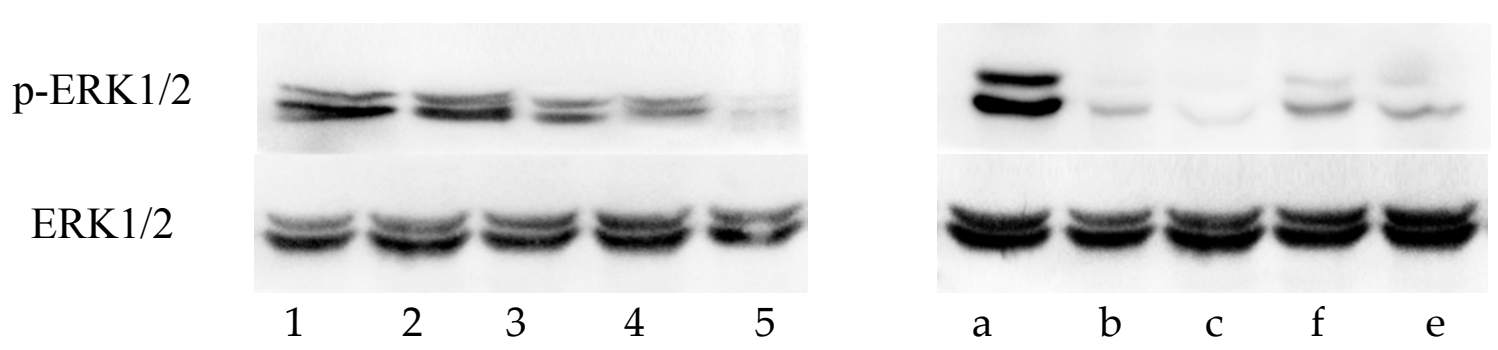

(C)

Figure 1. The amount of protein loaded in each lane is $100 \mu \mathrm{g}$. ERK1/2 was set as a loading control at the same time. ERK1/2, extracellular signal-regulated kinase 1 and 2; p-ERK1/2, phosphorylated of ERK1/2. (A) The Western blotting of p-ERK1/2 and ERK1/2 for Group C (the Chlamydia trachomatis group) at different time points. Lanes 1 to Lane 5 represent the time points of $0,12 \mathrm{~h}, 24 \mathrm{~h}, 36 \mathrm{~h}$, and $48 \mathrm{~h}$, respectively; (B) The Western blotting of p-ERK1/2 and ERK1/2 for four groups after $48 \mathrm{~h}$ of incubation. 1: Group C + A, the azithromycin-treated group. 2: Group C + V, the Vp1-treated group. 3: Group C, the Chlamydia trachomatis group. 4: Group M, the blank group (only McCoy cells); (C) The Western blotting of p-ERK1/2 and ERK1/2 for Group C $+\mathrm{V}$ and Group C + A during the incubation at different time points. Lanes 1 to Lane 5 represent the time points of $0,12 \mathrm{~h}, 24 \mathrm{~h}, 36 \mathrm{~h}$, and $48 \mathrm{~h}$ for Group $C+V$, respectively. Lanes a-e represent the time points of $0,12 h, 24 h, 36 h$, and $48 \mathrm{~h}$ for Group C + A, respectively. 


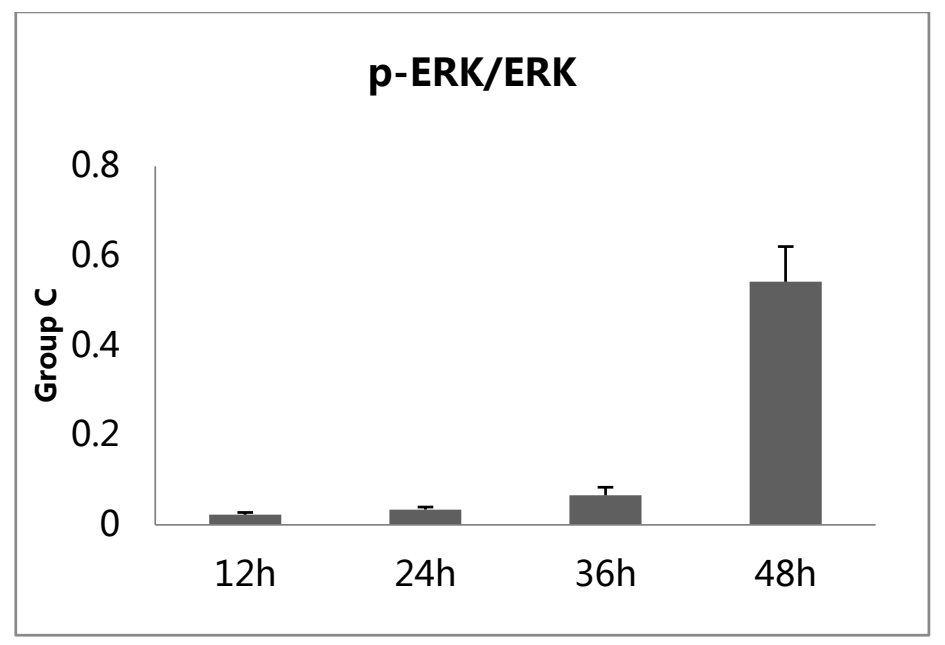

(A)

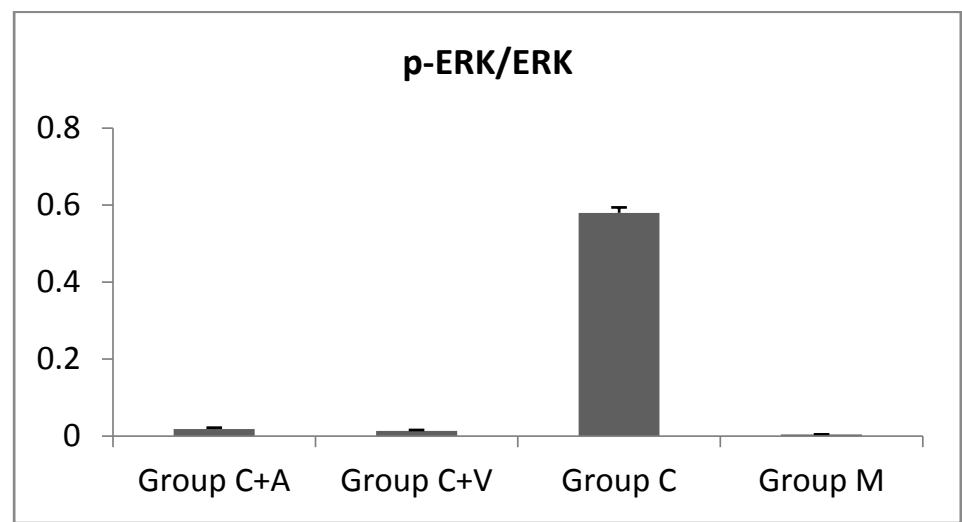

(B)

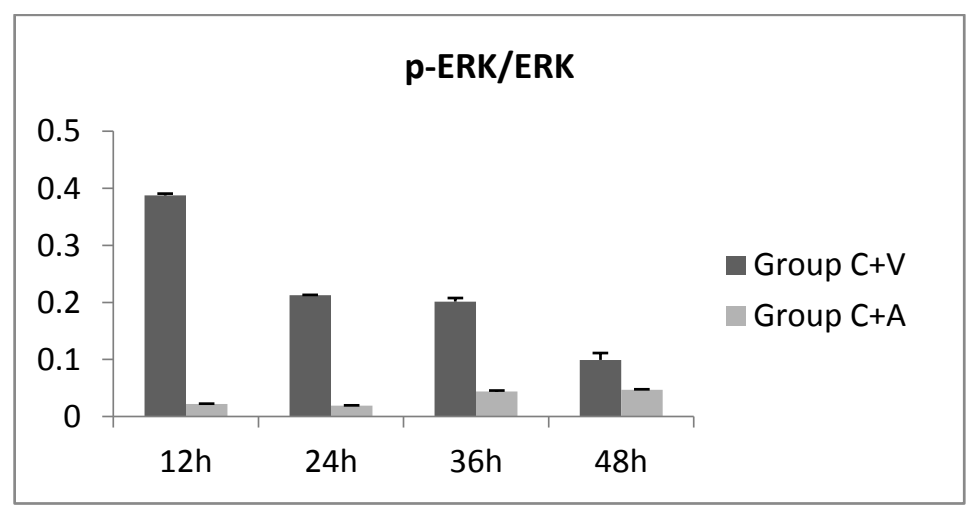

(C)

Figure 2. Grey value ratios of the ratio of $\mathrm{p}$-ERK1/2 to ERK1/2 expression (A). Group C at different time points. * Statistically significant difference between the value at $48 \mathrm{~h}$ and other three other time points by one-way ANOVA, LSD (Least Significant Difference) ( $p=0.000)$; (B) four groups after $48 \mathrm{~h}$ of incubation. * Statistically significant difference compared with Group C by the Paris $t$-test $(p=0.000)$; (C) Group $\mathrm{C}+\mathrm{V}$ and Group $\mathrm{C}+\mathrm{A}$ at different time points during the incubation. * Statistically significant difference compared with other three time points by one-way ANOVA, LSD $(p=0.000)$. ${ }^{*}$ Statistically significant difference compared with the value of $12 \mathrm{~h}(p=0.000)$. \# Statistically significant difference compared with the value of $12 \mathrm{~h}(p=0.028)$ and $24 \mathrm{~h}(p=0.017)$. \#\# Statistically significant difference compared with the value of $12 \mathrm{~h}(p=0.016)$ and $24 \mathrm{~h}(p=0.009)$. Group $\mathrm{C}+\mathrm{A}$, the azithromycin-treated group. Group C + V, the Vp1-treated group. Group C, the Chlamydia trachomatis group. Group M, the blank group (only McCoy cells). 
3.2. $p$-ERK1/2 Expression Was Reduced in the Vp1-and Azithromycin-Treated Cells as the Infection Rate Decreased

The number of inclusions decreased after $48 \mathrm{~h}$ of incubation in both Group C $+\mathrm{V}$ and Group C $+\mathrm{A}$ (Vp1-treated and azithromycin-treated infected cells, respectively) compared to that of Group C (C. trachomatis infection-only). The infection rate of Group C was over 90\%, with $63503.40 \pm 2216.13$ inclusions per well. The inhibition rate of Group C + V and Group C + A reached up to $76.48 \%$ (14933.00 \pm 1814.88 inclusions) and 64.45\% (22578.26 \pm 2583.70 inclusions), respectively (Figure 3).

Furthermore, significant differences in p-ERK1/2 expression levels were observed between the Chlamydia-infected groups $(\mathrm{C}+\mathrm{V}, \mathrm{C}+\mathrm{A}$, and $\mathrm{C})$ and the untreated control group (M) after $48 \mathrm{~h}$ of incubation (Figure 1B). The p-ERK1/2 expression level of Group C $(0.580 \pm 0.014)$ was much higher than that of Group M $(0.004 \pm 0.000)$, whereas those of Group C + V $(0.013 \pm 0.002)$ and Group C $+A$ $(0.018 \pm 0.004)$ were lower than that of Group C (Figure $2 B)$. This result indicates that, similar to azithromycin, the inhibition effect of Vp1 occurs through the MEK/ERK pathway.

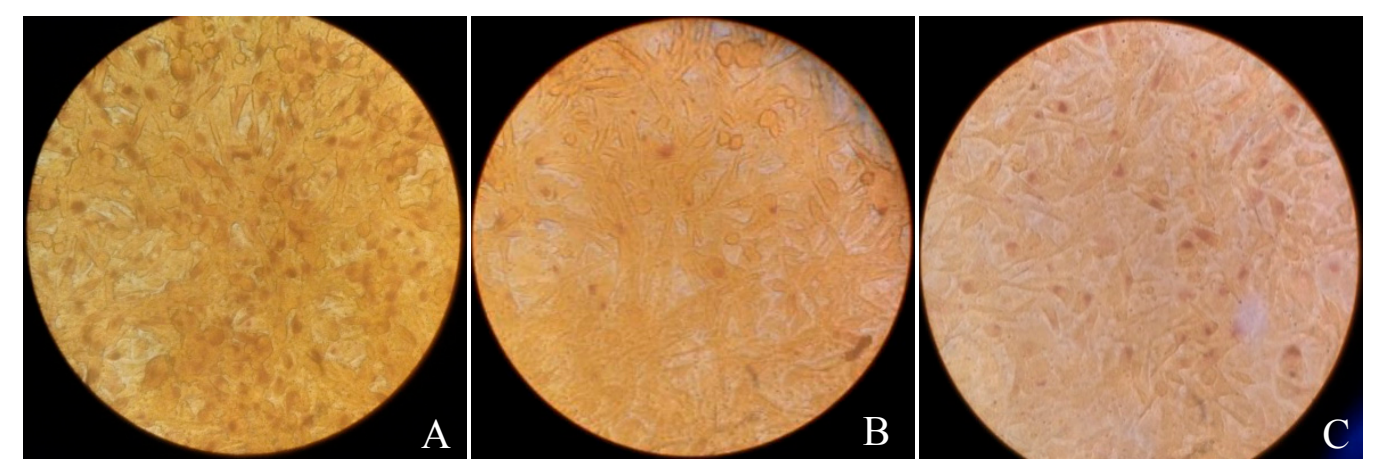

Figure 3. The inclusions viewed under the microscope $(\times 200)$ by iodine dye. The inclusions are stained dark brown and positioned decentered in the cells, whereas the normal cells are light-stained or uncolored. (A) the inclusions of Chlamydia trachomatis infection after $48 \mathrm{~h}$; (B) the inclusions of Vp1-treated Chlamydia trachomatis infection after $48 \mathrm{~h}$; (C) the inclusions of azithromycin-treated Chlamydia trachomatis infection after $48 \mathrm{~h}$.

\subsection{Vp1 Inhibits C. trachomatis in the Late Infection Period, Whereas Azithromycin Inhibits Growth in the Early Period}

The results described above clarified that $\mathrm{Vp} 1$ and azithromycin definitely inhibit the growth of Chlamydia trachomatis. The p-ERK1/2 levels of the cells were lower than the pre-intervention levels. Considering the changes over time in greater detail, in Group C + V, the p-ERK1/2 level gradually decreased from $12 \mathrm{~h}$ to $48 \mathrm{~h}$, whereas the decrease in Group C + A only started at $12 \mathrm{~h}$ to $24 \mathrm{~h}$ and was maintained at a low level until $48 \mathrm{~h}$ (Figures $1 \mathrm{C}$ and 2C). This result indicated that Vp1 exerts its effect on Chlamydia in the late infection period (36-48 h), whereas the inhibitory effect of azithromycin occurs in the early infection period (12-24 h).

\subsection{The ERK1 Gene Was Up-Regulated and the ERK2 Gene Was Downregulated after C. trachomatis Infection}

After $48 \mathrm{~h}$ of incubation, the relative ERK1 mRNA expression levels of cells in Groups C, C + V, and $\mathrm{C}+\mathrm{A}$ were all increased compared with that of Group M. The expression level of the Chlamydia infection group showed a 2.380-fold increase compared to the uninfected control cells. Furthermore, the ERK1 mRNA levels of the Vp1-treated and azithromycin-treated group showed a 1.659- and 1.915-fold increase compared to the control level, respectively. By contrast, the relative ERK2 mRNA expression levels of all three groups decreased after $48 \mathrm{~h}$ of incubation compared with the control group. The expression level of the Chlamydia group decreased by 0.883 -fold compared to the control, whereas the Vp1- and azithromycin-treated groups showed larger decreases of 0.827- and 0.797-fold compared to the control. 


\subsection{IL-8 and IL-1 Levels Decreased after Vp1 and Azithromycin Treatments}

The mean IL-8 production level increased when the cells were infected with Chlamydia compared to the uninfected control group (Group M) after $48 \mathrm{~h}$, whereas the IL-8 levels sharply decreased in the Vp1- and azithromycin-treated groups (Figure 4). From 0 to $48 \mathrm{~h}$ of incubation, the IL-8 levels of Group $\mathrm{M}$ were relatively stable, with a slightly early decrease and subsequent increase. However, Group C showed a considerable reduction in IL-8 production at $0-24 \mathrm{~h}$ and rapid growth from $24-48 \mathrm{~h}$. The Vp1-treated group showed a sharp increase at $0-12 \mathrm{~h}$ and a gradual decrease at $12-48 \mathrm{~h}$. The IL-8 level of the azithromycin-treated group increased at $0-24 \mathrm{~h}$, and decreased at $24-48 \mathrm{~h}$. Similar tendencies over time and between groups were observed for IL-1 production. The IL-1 level of Group C was increased comparing to that of the control, Group M. The IL-1 levels of the Vp1-and azithromycin-treated groups were reduced compared to those of Group C (Figure 4). During the incubation, IL-1 levels of Group M decreased from $0-12 \mathrm{~h}$ and were relatively stable from $12 \mathrm{~h}$ to $48 \mathrm{~h}$. Group $\mathrm{C}$ showed an increasing tendency from $12 \mathrm{~h}$ to $48 \mathrm{~h}$. The IL-1 level of the Vp1-treated group decreased from 0 to $24 \mathrm{~h}$, increased from $24 \mathrm{~h}$ to $36 \mathrm{~h}$, and further decreased from $36 \mathrm{~h}$ to $48 \mathrm{~h}$. Although the azithromycin-treated group showed a similar tendency to the Vp1-treated group, its peak was observed at $24 \mathrm{~h}$.
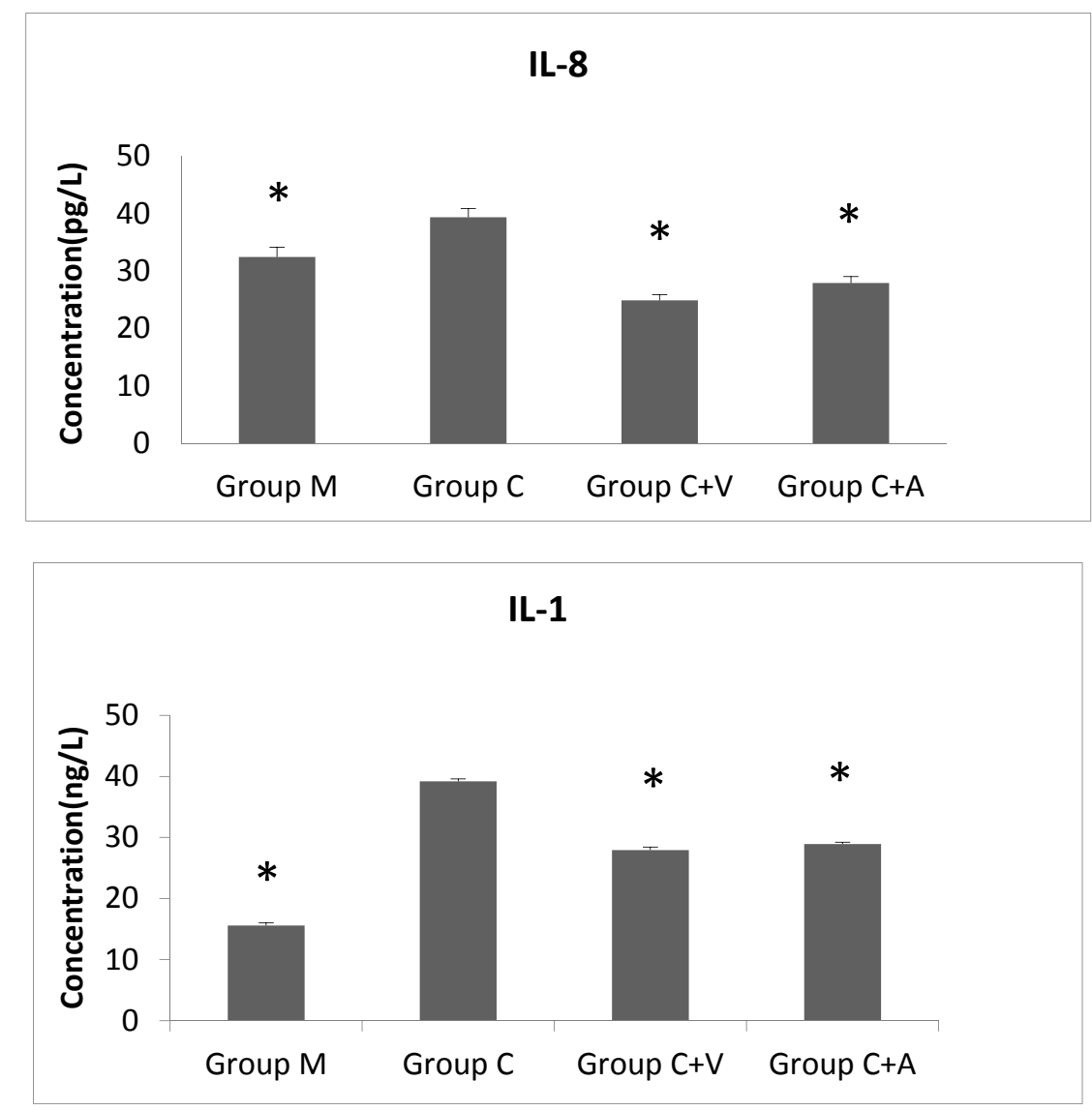

Figure 4. IL-8 and IL-1 production for four groups after $48 \mathrm{~h}$ of incubation. The experiment was repeated three times and the results are shown as mean with standard deviation. * Statistically significant difference compared with Group $C$ by the Paris $t$-test $(p=0.000)$. Group $C+A$, the azithromycin-treated group. Group C + V, the Vp1-treated group. Group C, the Chlamydia trachomatis group. Group M, the blank group (only McCoy cells). 


\section{Discussion}

C. trachomatis urogenital tract infection is a sexually transmitted disease that has become increasingly common and is a global health concern. The mechanisms of Chlamydia infection are closely related to inflammation-induced pathologic damage. Accordingly, the MAPK signaling pathways play a major role in the C. trachomatis infection, especially with respect to the growth and production of pro-inflammatory cytokines. To further understand the mechanisms of Chlamydia infection, it is important to investigate the mechanisms underlying the inhibition of $C$. trachomatis by the bacteriophage capsid protein Vp1. We here report the first attempt at exploring the relationship between $C$. trachomatis-infected cells treated with Vp1 and the MEK/ERK pathway at the protein and gene levels. Furthermore, we evaluated the effects of Vp1 treatment on the pro-inflammatory cytokines IL-8 and IL-1 to clarify the mechanism of inhibition.

First, we found that the p-ERK level increased gradually during C. trachomatis incubation with McCoy cells, which confirms Du et al. [8] in HeLa cells. In addition, Vp1 decreased the p-ERK level and the ERK1 and ERK2 mRNA expression levels, showing the same effects as azithromycin treatment. These results demonstrate that Vp1 and azithromycin have the same mechanism of Chlamydia inhibition by modulating the MEK/ERK pathway and minimizing ERK activation. The only difference was the effective time of the inhibition: the Vp1 is effective in the late infection period, while azithromycin was effective in the early period of Chlamydia growth.

Second, the ERK1 mRNA level was upregulated, while the ERK2 mRNA level was downregulated after C. trachomatis infection. Although the ERK1 and ERK2 genes have $90 \%$ sequence identity and share the same target upstream and downstream, they have some different biological functions. For example, ERK2 plays a key role in hepatocyte cell division, while ERK1 improves long-term hepatocyte survival [18]. In addition, specifically ERK2 but not ERK1 is required for the c-Met-paxillin signaling axis in HGF (Hepatocyte Growth Factor)-mediated motility [14]. The results of this study suggest that ERK1 might play a more important role in C. trachomatis growth than ERK2. However, confirmation of this hypothesis and the detailed mechanism await further investigation.

Finally, the pro-inflammatory cytokines IL-8 and IL-1 were found to increase after C. trachomatis infection, confirming previous research [11]. These two cytokines are directly induced by ERK pathways. In our study, production of these two cytokines also decreased after infected cells were treated with Vp1 and azithromycin compared with the untreated group. The IL-8 level of the Vp1-treated group decreased earlier than that of the azithromycin-treated group, while the IL-1 of the both groups showed an initial decrease (similar to the untreated infected cells), and then peaked at $24 \mathrm{~h}$ and $36 \mathrm{~h}$ before decreasing subsequently. The observed variations of IL-8 and IL-1 provide further understanding of the function of related pathways and their relationship to Chlamydia inhibition.

\section{Conclusions}

In conclusion, our research demonstrates that the mechanism of chlamydiaphage $\varphi$ CPG1 capsid protein Vp1 inhibiting C. trachomatis growth is associated with the MAPK pathway, via inhibition of the pro-inflammatory cytokines IL-8 and IL-1. Therefore, we have identified that bacteriophages may also influence their hosts via signaling transduction mediation and not only via bacteriolysis. Thus, we have uncovered a likely preliminary mechanism for how Vp1 inhibits C. trachomatis in cells. Nevertheless, the specific mechanisms determining the relationship between $\mathrm{Vp} 1$ and $C$. trachomatis are still under exploration.

Although azithromycin is still the first-choice recommended treatment for C. trachomatis [19], its clinical use has become less frequent because of acquired drug resistance. Although a chlamydia vaccine has been under exploration for several years and several candidate antigens have been screened, vaccine development remains a great challenge [20]. Therefore, the exploration of infection mechanisms offers new perspective for Chlamydia treatment. Bacteriophages have unique characteristics and show good potential as a clinical treatment to overcome antibiotic resistance [21]. The use of bacteriophages would minimize the chance of secondary infections because of their specific combination with hosts, 
which is a continued risk with antibiotics. In addition to their high specificity, the concentration at the site of infection can be assured so that side effects can be limited. Experts make efforts on phage transformation to get broad-spectrum, long-life and multi-function phages [22]. Therefore, the application of chlamydiaphages as a biotic therapy shows good future promise and warrants further exploration.

Acknowledgments: This research was supported by National Natural Science Foundation of China (Grant No.: 31370211).

Author Contributions: Yuanli Guo, Yuanjun Liu and Quanzhong Liu conceived and designed the experiments; Yuanli Guo and Rui Guo performed the experiments; Quan Zhou and Changgui Sun analyzed the data; Xinmei Zhang contributed materials and analysis tools; Yuanli Guo wrote the paper.

Conflicts of Interest: The authors declare no conflict of interest.

\section{References}

1. Centers for Disease Control and Prevention. 2014 Sexually Transmitted Diseases Surveillance. Available online: http:/ /www.cdc.gov/std/stats13/ (accessed on 10 February 2016).

2. Parish, W.L.; Laumann, E.O.; Cohen, M.S.; Suiming, P.; Heyi, Z.; Irving, H.; Tianfu, W.; Kwai Hang, N. Population-based study of chlamydial infection in China: A hidden epidemic. JAMA 2003, 289, 1265-1273. [CrossRef] [PubMed]

3. Hsia, R.C.; Ting, L.M.; Bavoil, P.M. Microvirus of Chlamydia psittaci strain guinea pig inclusion conjunctivitis: Isolation and molecular characterization. Microbiology 2000, 146, 1651-1660. [CrossRef] [PubMed]

4. Liu, B.L.; Everson, J.S.; Fane, B.; Giannikopoulou, P.; Vretou, E.; Lambden, P.R.; Clarke, I.N. Molecular characterization of a bacteriophage (Chp2) from Chlamydia psittaci. J. Virol. 2000, 74, 3464-3469. [CrossRef] [PubMed]

5. Read, T.D.; Brunham, R.C.; Shen, C.; Gill, S.R.; Heidelberg, J.F.; White, O.; Hickey, E.K.; Peterson, J.; Utterback, T.; Berry, K.; et al. Genome sequences of Chlamydia trachomatis MoPn and Chlamydia pneumoniae AR39. Nucleic Acids Res. 2000, 28, 1397-1406. [CrossRef] [PubMed]

6. Sliwa-Dominiak, J.; Suszynska, E.; Pawlikowska, M.; Deptula, W. Chlamydia bacteriophages. Arch. Microbiol. 2013, 195, 765-771. [CrossRef] [PubMed]

7. Liu, Y.-J.; Hou, S.-P.; Wei, J.-R.; Li, Y.; Qi, M.-L.; Wang, H.-P.; Liu, Q.-Z. The effect of Chlamydiaphage phiCPG1 capsid protein Vp1 on the Chlamydia trachomatis. Chin. J. Microbiol. Immunol. 2012, 32, 403-408. (In Chinese)

8. Jürgen, R.D.; Christina, G.; Hangxing, Y.; Katharina, W.; Otto, G.P.; Elisabeth, L.T.; Vera, F.B.; Eberhard, S. Persistent Chlamydia trachomatis infection of HeLa cells mediates apoptosis resistance through a Chlamydia protease-like activity factor-independent mechanism and induces high mobility group box 1 release. Infect. Immunity 2012, 80, 195-205.

9. Wen, C.; Pooja, S.; Youmin, Z.; Ding, C.; Zhongyu, L.; Guangming, Z. Intracellular interleukin-1 $\alpha$ mediates interleukin-8 production induced by Chlamydia trachomatis infection via a mechanism independent of type I interleukin-1 receptor. Infect. Immun. 2008, 76, 942-951.

10. Du, K.; Wang, F.-Y.; Huo, Z. Development of Chlamydia trachomatis is dependent on the MEK/ERK survival pathways. Life Sci. Res. 2010, 14, 424-426. (In Chinese)

11. Kavathas, P.B.; Boeras, C.M.; Mulla, M.J.; Abrahams, V.M. Nod1, but not the ASC inflammasome, contributes to induction of IL-1 $\beta$ secretion in human trophoblasts after sensing of Chlamydia trachomatis. Mucosal Immunol. 2013, 6, 235-243. [CrossRef] [PubMed]

12. Chen, F.; Cheng, W.; Zhang, S.; Zhong, G.; Ping, Y.U. Induction of IL-8 by Chlamydia trachomatis through MAPK pathway rather than NF-kB pathway. J. Cent. South Univ. 2010, 35, 307-313.

13. Wang, Y.X.; Li, L.-T. Detection of IL-2,IL-6 and IL-8 in Chlamydia trachomatis-infected reproductive tract in pregnant women. J. Shanxi Med. Univ. 2009, 05, 472-474. (In Chinese)

14. Patton, D.L.; Sweeney, Y.T.; Stamm, W.E. Significant reduction in inflammatory response in the macaque model of Chlamydial pelvic inflammatory disease with azithromycin treatment. J. Infect. Dis. 2005, 192, 129-135. [CrossRef] [PubMed] 
15. Srivastava, P.; Vardhan, H.; Bhengraj, A.R.; Jha, R.; Singh, L.C.; Salhan, S.; Mittal, A. Azithromycin treatment modulates the extracellular signal-regulated kinase mediated pathway and inhibits inflammatory cytokines and chemokines in epithelial cells from infertile women with recurrent chlamydia trachomatis infection. DNA Cell Biol. 2011, 30, 545-554. [CrossRef] [PubMed]

16. Liu, Q.-Z.; Yaom, W.-F.; Qi, M.-L.; Li, Y.; Wang, H.-P.; Tian, J.-Q. Cloning, expression and identification of chlamydial GPIC capsid Vp1 protein. Chin. J. Dermatol. 2006, 39, 714-716. (In Chinese)

17. Wang, X.; Seed, B. A PCR primer bank for quantitative gene expression analysis. Nucleic Acids Res. $2003,31$. [CrossRef]

18. Fremin, C.; Ezan, F.; Boisselier, P.; Bessard, A.; Pages, G.; Pouyssegur, J.; Baffet, G. ERK2 but not ERK1 plays a key role in hepatocyte replication: An RNAi-mediated ERK2 knockdown approach in wild-type and ERK1 null hepatocytes. Hepatology 2007, 45, 1035-1045. [CrossRef] [PubMed]

19. Potterat, J.J. 2010 European guideline for Chlamydia trachomatis infections: Recommending partner notification look-back periods. Int. J. STD AIDS 2011, 22. [CrossRef] [PubMed]

20. Hafner, L.M.; Wilson, D.P.; Timms, P. Development status and future prospects for a vaccine against Chlamydia trachomatis infection. Vaccine 2014, 32, 1563-1571. [CrossRef] [PubMed]

21. Golkar, Z.; Bagasra, O.; Pace, D.G. Bacteriophage therapy: A potential solution for the antibiotic resistance crisis. J. Infect. Dev. Ctries 2014, 8, 129-136. [CrossRef] [PubMed]

22. Elizabeth, K.; Daniel, D.V.; Guram, G.; Zemphira, A.; Lasha, G.; Sarah, K.; Abedon, S.T. Phage therapy in clinical practice: Treatment of human infections. Curr. Pharm. Biotechnol. 2010, 11, 69-86.

(C) 2016 by the authors; licensee MDPI, Basel, Switzerland. This article is an open access article distributed under the terms and conditions of the Creative Commons Attribution (CC-BY) license (http:/ / creativecommons.org/licenses/by/4.0/). 